Document downloaded from:

http://hdl.handle.net/10251/79248

This paper must be cited as:

Alves, N.; Mano, J.; Gómez Ribelles, JL.; Gómez-Tejedor, JA. (2004). Departure from the Vogel behaviour in the glass transition - thermally stimulated recovery, creep and dynamic mechanical analysis studies. Polymer. 45(3):1007-1017. doi:10.1016/j.polymer.2003.04.002.

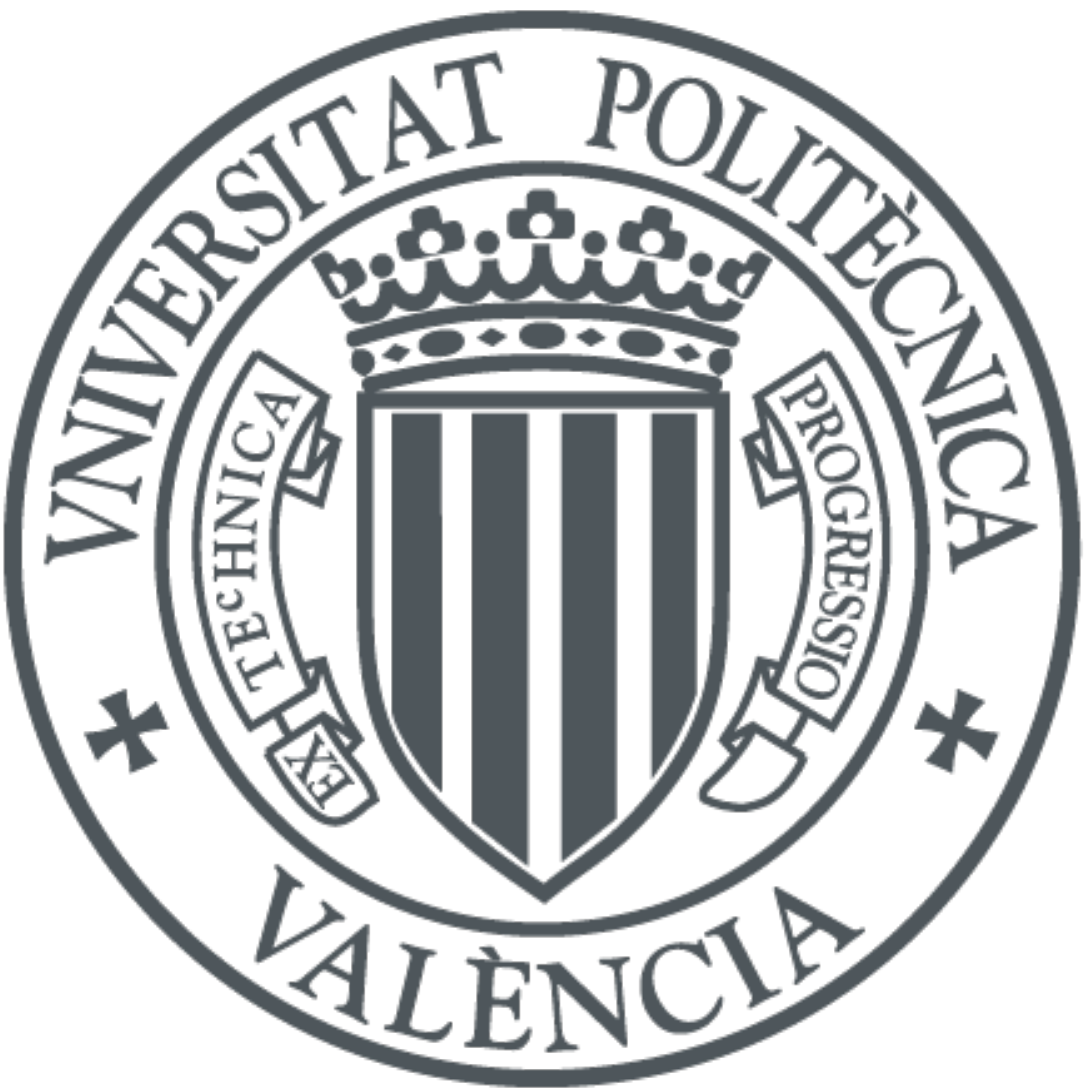

The final publication is available at

http://dx.doi. org/10.1016/j.polymer.2003.04.002

Copyright Elsevier

Additional Information 
N.M. Alves et al. / Polymer 45 (2004) 1007-1017

\title{
DEPARTURE FROM THE VOGEL BEHAVIOUR IN THE GLASS TRANSITION - THERMALLY STIMULATED RECOVERY, CREEP AND DYNAMIC MECHANICAL ANALYSIS STUDIES
}

\author{
N.M. Alves ${ }^{1}$, J.F. Mano ${ }^{1}$, J.L. Gómez Ribelles², J.A. Gómez Tejedor ${ }^{3}$ \\ ${ }^{1}$ Polymer Engineering Department, University of Minho, Campus of Azurém, 4800-058 Guimarães, Portugal. \\ ${ }^{2}$ Center for Biomaterials and Dept. of Applied Thermodynamics, Universidad Politécnica de Valencia, P.O. Box \\ 22012, E-46071 Valencia, Spain. \\ ${ }^{3}$ Center for Biomaterials and Dept. of Applied Physics, Universidad Politécnica de Valencia, Escuela \\ Universitaria de Informática, E-46022 Valencia, Spain.
}

\begin{abstract}
In this work the study of the dynamics of the segmental motions close to $T_{g}$ of a poly(methyl methacrylate), PMMA, network was analysed by distinct mechanical spectroscopy techniques. Three techniques were employed: dynamic mechanical analysis (DMA), creep and thermally stimulated recovery (TSR). The timetemperature superposition principle was applied to the DMA and creep results, and master curves were successfully constructed. A change from a Vogel to an Arrhenius behaviour was observed in these results. Above $T_{g}$ it was found a distinct temperature dependence for the retardation times calculated from creep and the relaxation times calculated from DMA. This unexpected behaviour was attributed to the merging of the $\alpha$ and the $\beta$ relaxations that occurs in PMMA systems. The activation energies $\left(E_{a}\right)$ were also calculated from DMA, creep and TSR experiments. Above $T_{g}$ the $E_{a}$ values obtained agreed very well for all the techniques. In addition, the fragility exhibited by this material was investigated by the mechanical spectroscopy techniques referred above and by differential scanning calorimetry (DSC). The obtained values of the fragility index $m$ indicated that the PMMA network is a kinetically fragile system. The thermodynamic manifestation of the fragility was also analysed.
\end{abstract}

Keywords: TSR, DMA, poly(methyl methacrylate), segmental relaxation, structural relaxation, fragility 
N.M. Alves et al. / Polymer 45 (2004) 1007-1017

\section{Introduction}

Many liquids (including covalent, ionic and metallic) are able to solidify into a disordered glassy structure upon cooling below their melting point, if crystallisation is prevented [1-3]. The glass transition corresponds to the freezing of the liquid-like mobility at the length scale of several molecular units. Microscopically, no structural change occurs during this process, and the glass maintains the same liquid-like structure [2,3]. In a more practical point of view, the glass transition is also associated to marked changes in thermodynamic derivatives properties, such as heat capacity and thermal expansivity, at temperatures around the glass transition temperature, $T_{g}$, so it is associated with the falling-out of equilibrium, when one goes from the liquid to the glassy state. The glass transition observed in the laboratory is not a phase transition, it is a kinetic event which depends upon the crossing of an experimental time scale and the time scales for the molecular rearrangements. The glassy state is unstable because a glass is continually relaxing towards equilibrium. We could consider the glass mechanically stable for practical purposes, if experimental observations are made on time scales fast compared to the molecular motions that allow the glass to relax, though it is out of thermodynamic equilibrium. The complex features of the glass transition [4] make it one of the most difficult and unsolved problems in solid state physics and much attention has been devoted in looking for adequate manners of highlighting the universal features of glass-forming systems. The fragility concept is one of the attempts to systematise this issue, first introduced by Angell [5]. It is a measure of the rate at which the structural characteristic times, $\tau$ (or related properties, such as the shear viscosity $\eta$ ) decreases with increasing temperature around $T_{g}$ when plotted on a normalised $T_{g} / T$ plot. Here, the glass transition temperature is often defined either as the temperature at which $\tau \sim 100$ s or $\eta \sim 10^{12}$ Pa.s. Such $\log \tau$ (or $\log \eta$ ) vs $T_{g} / T$ plots were popularised by Angell, who used them to correlate with transport properties of glass-formers [5]. As summarised by Huang and McKenna, the dynamic fragility has been related with the nonexponentiality of the structural relaxation function, the chemical structure of polymers, the structural recovery in the glassy state and the vibrational motions [6]. The terms "strong" and "fragile" are now familiar terms in this context. "Strong" liquids are those characterised by follow a near Arrhenius transport behaviour, $\tau=\tau_{0} \exp \left(E_{a} / R T\right)$, and tend to be of tetrahedral network structure. On the other hand, ionic or van der Waals liquids are usually "fragile", and connoted to the sensitivity to temperature of their structure. Here, the Angell plots present a large curvature, being the apparent activation energy at lower temperatures (near $T_{g}$ ) usually high (sometimes higher than the vaporisation energy). Thermodynamically, strong liquids show small $\Delta C_{p}$ (difference of heat capacity between liquid and glass). This difference is much higher in fragile liquids.

For an understanding of the vitrification process the strong-fragile classification has been proven to be very relevant. It provides the basis for the interpretation of vitrification in terms of fundamental thermodynamic quantities from which other material properties can be predicted. However, the molecular origin of fragility is far to be completely understood. A major problem impeding progress is the own quantification of fragility, which has often found to depend upon the technique used for a given material [7] (e.g. dielectric, calorimetric, shear 
N.M. Alves et al. / Polymer 45 (2004) 1007-1017

compliance, shear and tensile moduli and quasielastic light scattering experiments). The aim of this work is to analyse the influence of the mechanical spectroscopy technique employed in the study of the dynamics of segmental motions close to $T_{g}$. Three techniques will be employed in this work: i) dynamic mechanical analysis (DMA), ii) isothermal creep experiments and iii) thermally stimulated recovery (TSR).

In the TSR technique the change of strain in a previously thermal/mechanical treated sample is recorded during a temperature scanning program. TSR has been shown to be a very sensitive technique in polymer systems, including composites, semicrystalline polymers, inter-penetrated polymer networks and thermosets, especially if combined with DMA [8-13]. By means of the Thermal Sampling procedure (TS), the TSR technique also offers the possibility of experimentally decompose a complex process, characterised by a distribution of characteristic times, into its quasi-individual components. This procedure enables the analysis of the fine structure of the TSR global spectra.

The relatively low equivalent frequency of TSR $\left(\sim 10^{-3}-10^{-2} \mathrm{~Hz}\right.$ [14]), compared with typical Dynamic Mechanical Analysis (DMA) experiments, warrants a good sensitivity on studying complex relaxation phenomena. As the time scales associated to TSR measurements are similar to those used in the description of fragility ( $\tau \sim 100 \mathrm{~s}$ ), this technique may be directly applied in such analysis. It should be also pointed out that the equivalent dielectric technique of TSR is the thermally stimulated depolarisation currents technique (TSDC), where a static electric field is applied instead of a mechanical load and the polarisation is monitored instead of the strain. TSDC was already used in the particular issue of quantifying the fragility, both in low molecular weight or polymeric materials $[15,16]$.

In this work the glass transition dynamics studied by DMA, creep and TSR will be compared for a poly(methyl methacrylate) network. Additionally differential scanning calorimetry (DSC) was also used in samples with different thermal histories to obtain further information about the fragility exhibited by this material.

\section{Experimental Section}

\subsection{Material}

The studied material was a copolymer of methyl methacrylate (Aldrich, 99\% pure) and ethylene glycol dimethacrylate (Aldrich, 98\% pure) containing 5\% of the latter. The polymer network was synthesised by free radical addition polymerisation using as photoinitiator $0.13 \%$ by weight of benzoin (Scharlau, $98 \%$ pure). The sample was synthesised between two glass plates to form a sheet of approximately $0.5 \mathrm{~mm}$ thick. The reason to work with a polymer network is to prevent permanent flow above $\mathrm{T}_{\mathrm{g}}$, allowing to perform both DMA, creep and TSR experiments across the glass-transition. The monomer, crosslinking agent and initiator were used as received without further purification. Polymerisation took place at room temperature for $24 \mathrm{~h}$ under UV radiation. The low molecular weight substances remaining in the sample after polymerisation were extracted with boiling ethanol for $24 \mathrm{~h}$ and then dried in vacuo at $70^{\circ}$ for several weeks until the weight remained constant. Finally the sample was dried in vacuo at $180^{\circ} \mathrm{C}$ for $1 \mathrm{~h}$ in order to eliminate possible residues that still remained in the sample. 


\subsection{Techniques}

The dynamic mechanical analysis (DMA) was performed with a Seiko DMS210 equipment in the extension mode, in isothermal conditions, at different temperatures, from $109.6{ }^{\circ} \mathrm{C}$ to $156.4{ }^{\circ} \mathrm{C}$ every $2{ }^{\circ} \mathrm{C}$. At each temperature the frequency was scanned from 0.01 to $20 \mathrm{~Hz}$.

Creep experiments were made with a Seiko TMA/6000 equipment in the extension mode. The maximum deformation of the sample was $1 \%$. The stress was applied within less than $10 \mathrm{~s}$ before time 0 and then maintained constant for a time between 36 and 112 minutes in isothermal conditions. The sample length was measured as a function of time at equal time intervals in logarithmic scale, and then, the creep compliance $D(t)$ was calculated. After creep, the stress was removed, and the sample recovered for a time twice the creep time. The experiment has been carried out at different temperatures, from $122.9^{\circ} \mathrm{C}$ to $150.5^{\circ} \mathrm{C}$ every $1{ }^{\circ} \mathrm{C}$.

Thermally stimulated recovery (TSR) experiments were carried out in a DMA7e Perkin-Elmer analyser with controlled cooling accessory in the extension mode. Continuous flux of high purity helium (flow rate of $\sim 28$ $\mathrm{cm}^{3} \mathrm{~min}^{-1}$ ) was used to improve heat transfer throughout the sample surroundings during the experiments.

At least two kinds of experiments can be performed with the TSR technique: the TSR global and the thermal sampling (or windowing) experiments - TS. In both types of experiments a static stress, $\sigma_{0}$, is applied during an isothermal period, $t_{\sigma}$ at a creep temperature $T_{\sigma}$, and during a temperature program at constant rate between $T_{\sigma}$ and $T_{\sigma^{-}} \Delta T_{w}$. Without any stress the strain is then partially recovered during an isothermal period at $T_{\sigma^{-}} \Delta T_{w}$ followed by a cooling down to $T_{0}$. Finally, the strain is measured, as a function of temperature, during a heating at constant rate up to a temperature well above $T_{\sigma}$. The difference between both experiments is that, in a TSR global experiment $T_{\sigma^{-}} \Delta T_{w}=T_{0}$, whereas in a TS experiment $\Delta T_{w} \sim 3{ }^{\circ} \mathrm{C}$ and $T_{0}<<T_{\sigma}$. In all the experiments carried out in this work we used a heating rate of $4 \mathrm{~K} \cdot \mathrm{min}^{-1}$ and for the TS experiments $\Delta T_{w}=3{ }^{\circ} \mathrm{C}$. More details about these tests, including more experimental details can be found elsewhere $[11,12]$.

In TS experiments the recovery measured during the heating scan is due to the molecular groups that were activated during the application of a static stress $\sigma_{0}$, which are those having retardation times at $T_{\sigma}$ around a certain characteristic time $\tau_{\sigma}$ which depends on the period of time in which the charge is applied. Thus, the TS experiment allows to resolve the complex retardation times spectrum in nearly elementary mechanisms. On the contrary, in the TSR global experiments the complex nature of the relaxation is studied because all the conformational motions with relaxation times around $\tau_{\sigma}$ between $T_{\sigma}$ and $T_{0}$ are activated. The ensemble of the TS experiments within the glass transition region gives an overall picture of the processes associated to the relaxation as probed at low frequencies.

The differential scanning calorimetry, DSC, experiments were carried out in a Perkin-Elmer DSC7 differential scanning calorimeter with controlled cooling accessory. The temperature of the equipment was calibrated with indium and lead standards and for the heat flow calibration only the same indium sample was used. All calibrations were carried out during heating, at $10^{\circ} \mathrm{C} / \mathrm{min}$. A single $10.295 \mathrm{mg}$ polymer sample, sealed in an 
N.M. Alves et al. / Polymer 45 (2004) 1007-1017

aluminium pan, was used for all DSC experiments. The DSC experiments were conducted as following: i) The sample was heated to above $T_{g}\left(121.4{ }^{\circ} \mathrm{C}\right)$ in order to erase any thermal history; ii) the sample is then cooled to $50{ }^{\circ} \mathrm{C}$ at different scanning rates, $q_{c}=\mathrm{d} T / \mathrm{d} t$ (between 0.5 and $40{ }^{\circ} \mathrm{C} \cdot \mathrm{min}^{-1}$ iii); data is subsequently collected during an heating ramp at $10^{\circ} \mathrm{C} / \mathrm{min}$ up to $180^{\circ} \mathrm{C}$.

\section{Results and Discussion}

\subsection{DMA and creep data}

Isothermal frequency DMA scans around $T_{g}$ are shown in Figure 1a (storage modulus, $E^{\prime}$ ) and Figure 1b (loss factor, $\tan \delta)$. The creep compliance $\mathrm{D}(\mathrm{t})$ at several temperatures is shown in Figure 2.

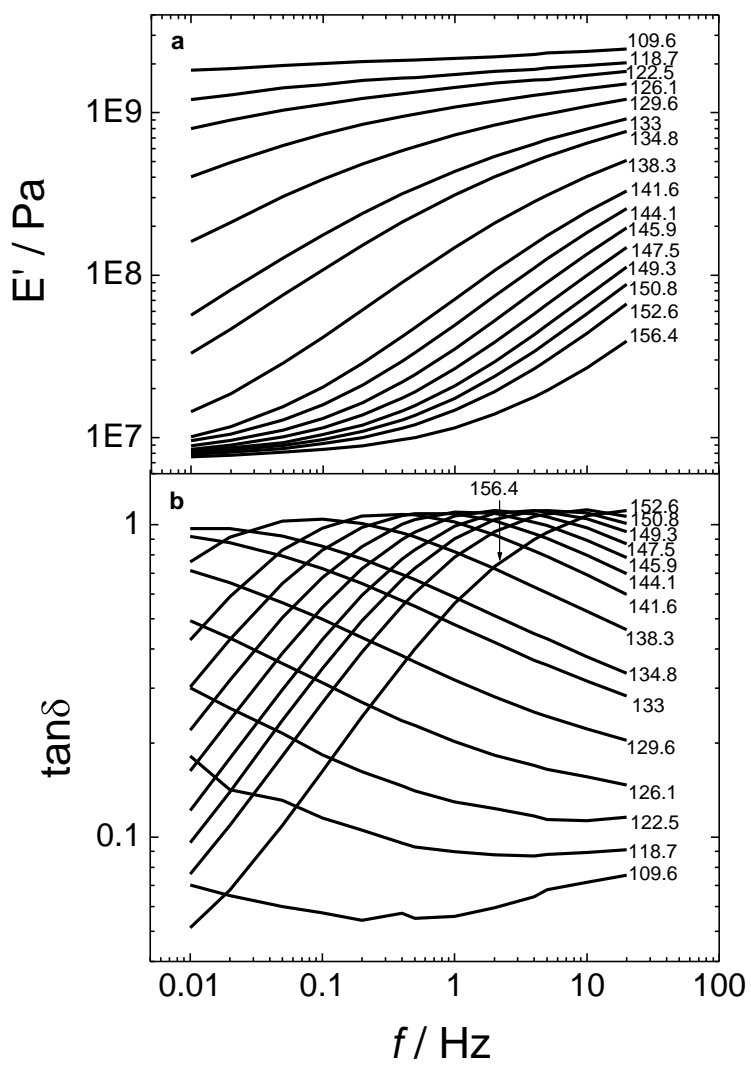

Figure 1 - DMA isothermal curves on the studied PMMA at different temperatures (in the graphics). a) Storage modulus ( $\left.E^{\prime}\right)$ vs frequency. b) Loss factor $(\tan \delta)$ vs frequency. For clarity, not all measured curves are presented.

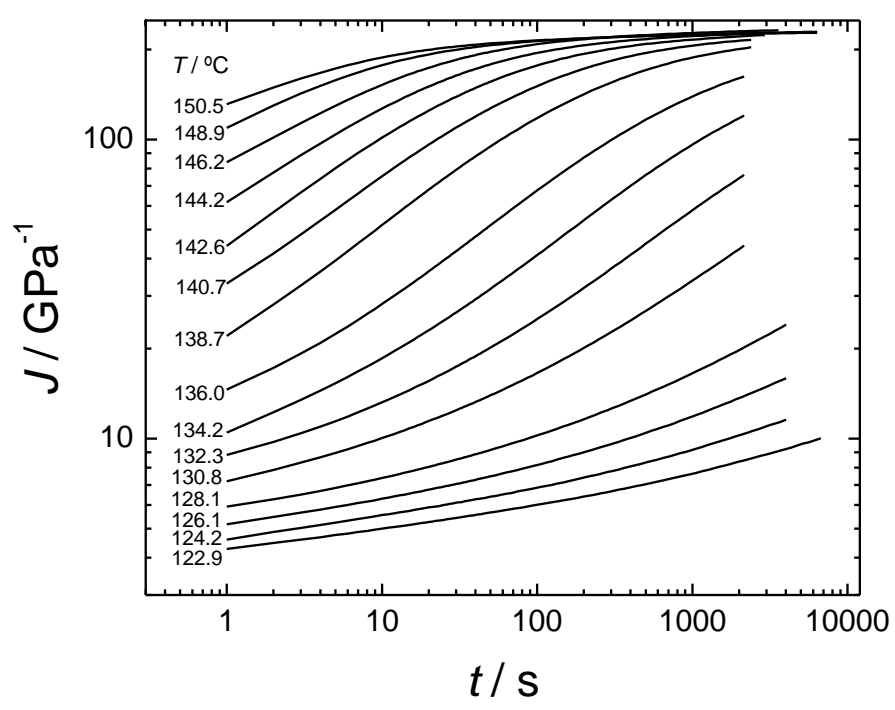

Figure 2 - Creep isothermal curves on the studied PMMA at different temperatures (in the graphics). For clarity, only isothermal every $2{ }^{\circ} \mathrm{C}$ has been plotted. 
N.M. Alves et al. / Polymer 45 (2004) 1007-1017

It is still under discussion if a time-temperature superposition principle [17] is accurately fulfilled by any of these series of results. According to other authors the thermorheological simplicity is no longer valid for compliances above $\sim 10^{-7} \mathrm{~Pa}^{-1}[4,18,19]$. In the previously referred works greater compliances are related to modified Rouse-like modes and in this case the construction of master curves by simple (i.e. just horizontal) shifts would be no longer valid. In addition the construction of master curves for PMMA could be not so straightforward because exists the additional problem of some degree of overlap between the $\alpha$ and $\beta$-processes [20] even in a crosslinked material, being the $\beta$-process especially broad.

In our work, master curves for the storage modulus $\left(E^{\prime}\right)$ and loss factor $(\tan \delta)$ were successfully obtained by simple shifting of the isothermal results along the frequency scale, according to the time-temperature superposition principle.

Figure 3 shows the plots of $E^{\prime}$ and $\tan \delta$ as a function of reduced frequency $f \mathrm{a}_{T}$, for the reference temperature $T_{\text {ref }}=147.5^{\circ} \mathrm{C}$.

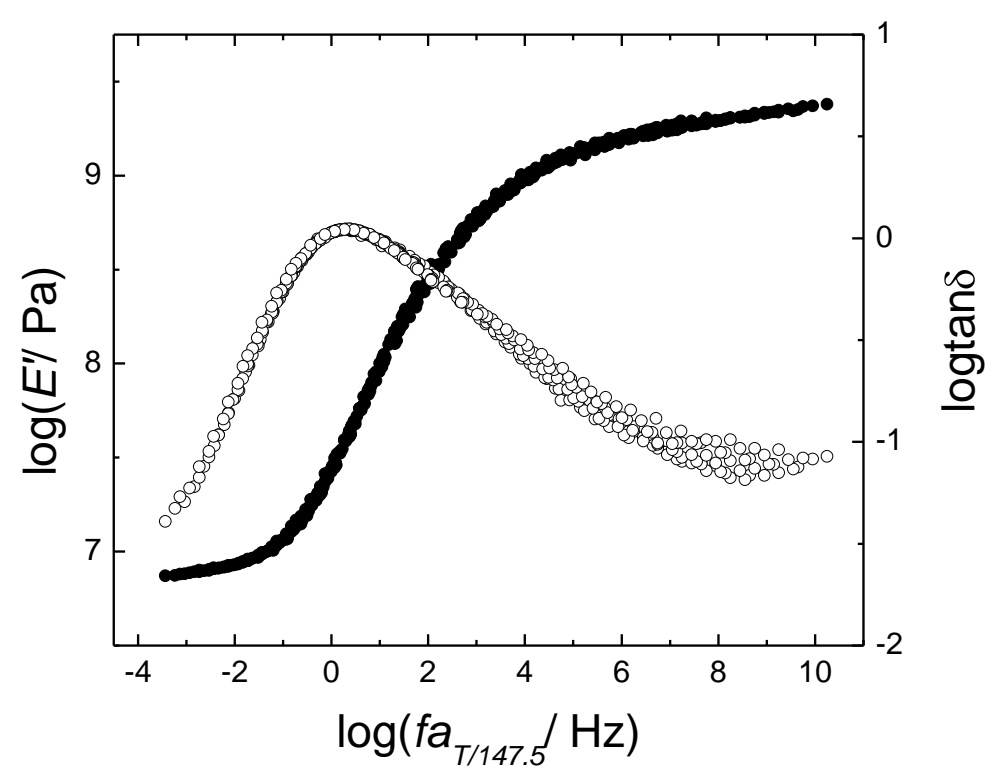

Figure 3 - Master curves for storage modulus (filled circles) and for loss factor (open circles) obtained from horizontal displacements of the curves of Figure 1, for a reference temperature of $147.5^{\circ} \mathrm{C}$.

Also a master curve for creep compliance was constructed (Figure 4), as a function of time, for the reference temperature $T_{r e f}=136.0^{\circ} \mathrm{C}$. As for the case of DMA results the master curve was obtained by simple shifting of the isothermal results along the log time scale. It should be pointed that in figures 3 and 4 all measured data were employed. 


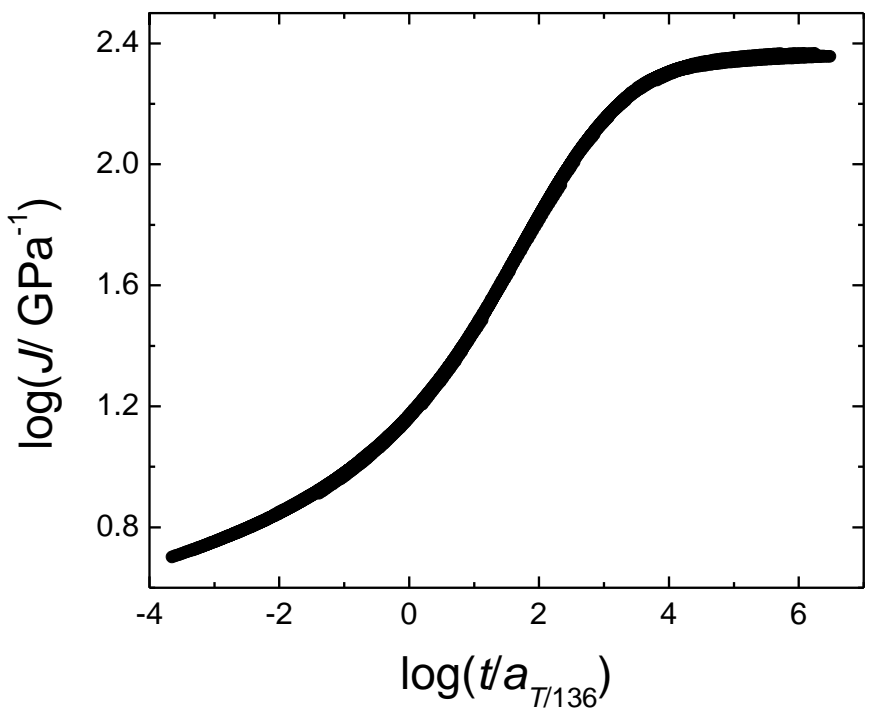

Figure 4 - Master curve for creep compliance obtained from horizontal displacements of the curves of Figure 2, for a reference temperature of $136.0^{\circ} \mathrm{C}$.

In principle, in order to account for the change in polymer density between $T$ and $T^{*}$, the vertical shift $\rho T / \rho^{*} T^{*}$ should also be applied to each curve of Figure 3 and 4 [17]. However, in the studied cases this correction seemed to be negligible.

The shift factors

$$
\log a_{T}=\frac{\tau(T)}{\tau(\text { Tref })}
$$

for both techniques are represented in Figure 5 against temperature. 


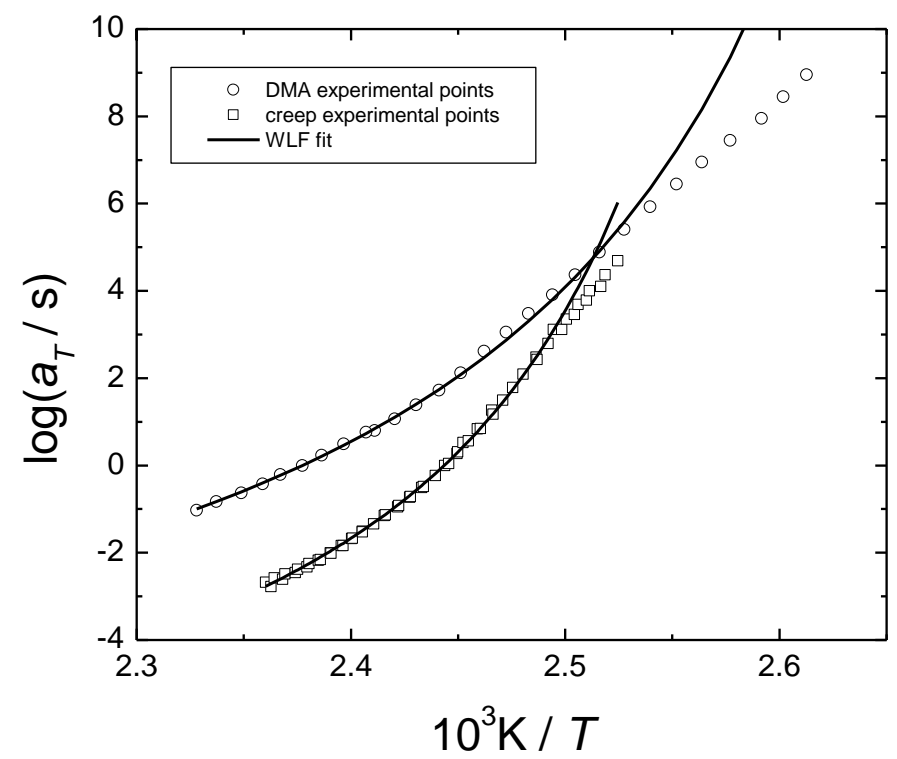

Figure 5- Temperature dependence of the shift factors for the DMA and creep master curves. The high temperature ranges of the data were fitted according to the WLF equation.

For the DMA master curves these shift factors associated with the two curves were found to be the same. $\tau(T)$ is a relaxation time $\tau_{E}$ in the case of DMA results and a retardation time $\tau_{D}$ in the case of creep experiments. The relationship between $\tau_{E}$ and $\tau_{D}$ comes from the relationship existing between modulus, $E(t)$, and compliance, $D(t)$, in the linear viscoelastic regime:

$$
\int_{0} D\left(t^{\prime}\right) E\left(t-t^{\prime}\right) d t^{\prime}=t
$$

For the case of a single relaxation time model, equation 2 gives the following relation between $\tau_{D}$ and $\tau_{E}$

$$
\frac{\tau_{E}}{\tau_{D}}=\frac{D_{U}}{D_{R}}
$$

where $D_{U}$ and $D_{R}$ are the limits of the compliance when the time tends to zero and infinity respectively. In this case this means that $\tau_{D}$ is more than three decades longer than $\tau_{E}$ In the case of a distribution of relaxation times the difference between the times that characterise the position of the distribution in the time axis in creep and stress relaxation process departs from equation (3) but still differences are very important. This problem will be 
N.M. Alves et al. / Polymer 45 (2004) 1007-1017

analysed in detail elsewhere. In this work we will make use only of the derivatives of the logarithm of the relaxation times with reciprocal of temperature and, as a consequence the absolute values of the relaxation times are not relevant for our discussion.

The shift factors above $T_{g}$, were successfully described by the WLF equation [21]:

$\log a_{T}=-\frac{C_{1}\left(T-T_{r e f}\right)}{C_{2}+\left(T-T_{r e f}\right)}$

where $C_{1}$ and $C_{2}$ depend on the material and on $T_{\text {ref. }}$. This expression is usually valid for polymers over the temperature range $T_{g}<T<T_{g}+100^{\circ} \mathrm{C}$ (where $T_{g}$ is the glass transition temperature) and when $T_{\text {ref }}$ is identified with $T_{g}$, it was seen that $C_{1}$ and $C_{2}$ assume "universal" values close to 17.44 and $51.6{ }^{\circ} \mathrm{C}$, respectively [21]. Equation 4 is equivalent to the Vogel-Fulcher-Tamman-Hesse equation (VFTH) [22-24],

$\tau(T)=\tau_{0} \exp \frac{B}{T-T_{0}}, T_{0}<T_{g}$

where $\tau_{0}$ is a pre-exponential factor and $B$ and $T_{0}$ are specific adjustable parameters. The WLF and VFTH equations are the most frequently applied for describing the non-Arrhenius behaviour, although exists a wide variety of mathematical representations (see for example references [25-29]). In the VFTH equation $T_{0}$ is a diverging temperature, implying the physical impossibility of configurational changes in the solid (the configurational entropy, $S_{c}$, tends to 0 at that temperature), close to the so-called Kauzmann temperature and $D=B / T_{0}$ is the strength parameter, which can be related with fragility: a $D$ value $(>30)$ represents a "strong" behaviour and a low $D$ values $(<30)$ is for a "fragile" behaviour. The WLF and VFTH parameters are related by $C_{1}=B / 2.303 C_{r e f}-T_{0}$, and $C_{2}=T_{r e f}-T_{0}[17]$.

The data in Figure 5 above $T_{g}$ were fitted with the WLF equation. The obtained parameters were $C_{1}=7.6$ and $C_{2}=59.1^{\circ} \mathrm{C}$ with $T_{r e f}=147.5^{\circ} \mathrm{C}$ for the DMA results and $C_{1}=6.08$ and $C_{2}=49.7^{\circ} \mathrm{C}$ with $T_{r e f}=136.0^{\circ} \mathrm{C}$ for creep. However, a deviation from the WLF line is observed at low temperatures, around the glass transition. Below $T_{g}$ the Arrhenius diagram tends to adopt a linear behaviour, but to see this behaviour in a more clear way data at lower temperature would be necessary. Obviously, the same trend should also be observed in the temperature dependence of the relaxation times, as $\log \tau(T)=\log a_{T}+\log \tau\left(T_{r e f}\right)$. This behaviour was also found for other authors [30-32], and has also been detected in dielectric relaxation spectroscopy results, although in a much narrow frequency range [33-35].

A robust theoretical rationalisation of the WLF (or VFTH) equation can be achieved with the Adam-Gibbs theory. According to the Adam-Gibbs model [36] relaxation is accomplished by rearrangements within "cooperatively rearranging regions" (CRRs), defined as "the smallest region that can undergo a transition to a 
N.M. Alves et al. / Polymer 45 (2004) 1007-1017

new configurational state without a requisite simultaneous configurational change on and outside its boundary". The length scale of CRRs increases with decreasing temperature although very slowly until close to the Kauzmann temperature. The size of CRRs, i.e. the characteristic length of glass transition, is at the present evaluated between $1-5 \mathrm{~nm}$ [37,38]. The temperature dependence of the CRRs size allows to relate the structural relaxation time with both the temperature and the configurational entropy, $S_{c}$ :

$$
\tau(T)=\tau_{0} \exp \left(C / T S_{C}(T)\right)
$$

where $\tau_{0}$ and $C$ are constants. $S_{c}$ can be calculated from the excess heat capacity, $\Delta C_{p}=C_{p}\left(\right.$ liquid)- $C_{p}($ glass):

$$
S_{c}(T)=\int_{T_{0}}^{T} \frac{\Delta C_{p}}{T^{\prime}} d T^{\prime}
$$

where $T_{0}$ is the temperature at which $S_{c}$ extrapolates to zero.

The curvature of $\tau(T)$ in the Ahrrenius diagram comes form the dependence of the configurational entropy with temperature. Different equations for the configurational heat capacity $\Delta C_{p}(T)$ (a constant, a linear temperature dependence or an hyperbolic variation on $\Delta C_{p}$ with temperature, $\Delta C_{p}=\alpha / T$ are the expressions most used $\left.[21,39]\right)$ yield different analytical forms of $S_{c}(T)$, all of them keeping the characteristic curvature. The glass transition produces a collapse in the conformational mobility and, as a consequence a smaller temperature dependence of $S_{c}$. When a polymer sample is cooled down from equilibrium across the glass transition, the configurational entropy tends to be independent on temperature in the glassy state with a value $S_{c}{ }^{g}$ which depends on the cooling rate. This means that in the glassy state, at temperatures below $T_{g}$, the relaxation times tend to the equation

$$
\tau(T)=\tau_{0} \exp \left(C / T S_{c}^{g}(T)\right)
$$

what corresponds to the Ahrrenius behaviour with an apparent activation energy $E_{a}{ }^{g}=C / S_{c}{ }^{g}$. This value is smaller at any temperature than the equilibrium one. The deviation from the equilibrium WLF line to the Ahrrenius behaviour implies a decrease in the slope of the $\log a_{T} v s .1 / T$ line as can be seen in figure 5 .

As seen in Figure 5 the slope of the $\log a_{T}$ vs $1 / T$ line at temperatures above $T_{g}$ is higher for the retardation time calculated from creep experiments than for the relaxation times calculated from DMA results. This means that the value of the parameter $B$ in the VFTH equation is not the same for both lines $\left(B_{D}=691.6 \mathrm{~K}\right.$ for the compliance and $B_{E}=1034.4 \mathrm{~K}$ for modulus). On the contrary in poly(propylene glycol) and poly(vinyl acetate) the same temperature dependence of relaxation and retardation times was found [40]. In the case of the PMMA network of this work the difference in slope is high enough to have no doubt of the experimental result. A similar result was found for a PMMA in reference [32]. The overlap of the main $\alpha$ relaxation and the secondary 
N.M. Alves et al. / Polymer 45 (2004) 1007-1017

$\beta$ relaxation can be the cause of this peculiar behaviour, in the case of PMMA the $\beta$ relaxation has been associated with the rotation of the esther group relatively to the C-C bond by which this group is linked to the main chain [41,42] and has an apparent activation energy much smaller than that of the main relaxation [43]. With increasing temperature and frequency the $\alpha$ and $\beta$ processes approach each other [44] and tend to coalesce originating an unique process called $\alpha \beta$ or $a$ process. Williams $[45,46]$ defined this process as a new relaxation process where the motions associated to the $\alpha$ and $\beta$ relaxations influence each other and not just a mere superposition of two relaxation mechanisms. However the $\alpha \beta$ process is not totally clarified, namely it is not so straightforward what happens in the region of frequencies and temperatures where occurs the separation of the two processes (splitting region) [47]. This problem is related to the glass transition dynamics because almost all the details of what the concept cooperativity means are unknown. The $\alpha \beta$ process of poly(n-alkyl methacrylates) was studied some years ago and several scenarios for the splitting region were proposed not only for these materials but for any material [47]. From this work it is inferred that the behaviour in the splitting region is much more complex than what may be expected, and the way the splitting occurs and the character of the local process could vary considerably in the splitting zone (two examples: the $\beta$ relaxation can be seen as a precursor process of the $\alpha$ relaxation, or instead the $\alpha$ relaxation process is continuous and the motions associated to the $\beta$ relaxation can only occur at temperatures much lower than $T_{g}$ ).

In the frequency dependence of the elastic modulus (Figure 3) the main relaxation corresponds to the steepest $E$, step, at the lowest frequencies and for $E^{\prime}$ values below around $10^{8.5} \mathrm{~Pa}$, and the influence of the $\beta$ relaxation seems to be more important in the high frequency part of the relaxation spectrum. In this frequency range $E$, increases smoothly with frequency. So, the presence of the secondary relaxation must play an important role in the shifts necessary to superpose the different isotherms to form the master curve.

The situation in the creep compliance $D(t)$, Figure 4, is similar but now the part of the curve in which the secondary relaxation has the highest influence is that corresponding to the smaller values of $D$ and probably its influence in the global process is smaller. In fact if modulus and compliance were represented in a linear scale against frequency and time respectively, the former would be representative mainly of the secondary relaxation while the letter would be mainly representative of the $\alpha$ relaxation. The consequence is the difference in the slope of the diagram shown in Figure 5. The change from VFTH to Ahrrenius appears in both lines at temperatures close to each other as will be discussed below after presenting the TSR results.

\subsection{TSR results}

Only thermal sampling (TS) experiments are reported in this work, at different $T_{\sigma}$ around the glass transition of the studied PMMA. Creep temperatures between 71.5 and $148.5^{\circ} \mathrm{C}$ were used. Figure 6 shows typical results, where the curves are shifted to higher temperatures as $T_{\sigma}$ increases and the low temperature plateau presents higher $\varepsilon$ values as $T_{\sigma}$ increases. This fact was explained elsewhere [12]. 


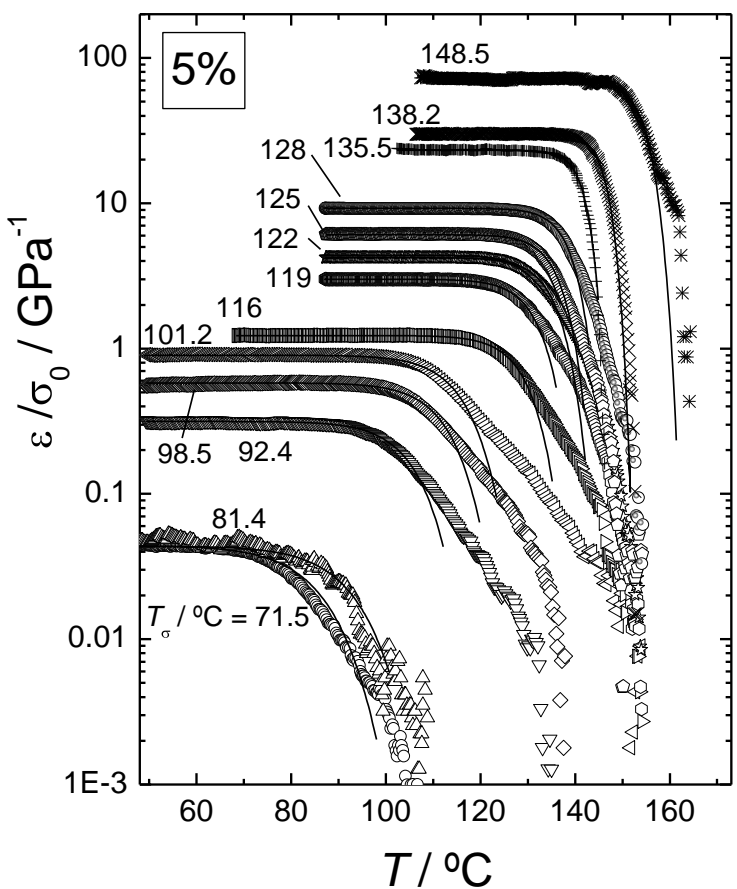

Figure 6- TS results on the studied PMMA in the glass transition region, obtained at different creep temperatures $T_{\sigma}$ (in the graphics). The solid lines are the simulated curves with the thermokinetic parameters obtained from the Arrhenius fitting of the $\tau(T)$ results such those presented in Figure 7.

Each TS curve contains information about the molecular mobility that mainly occur at the corresponding $T_{\sigma}$ for a time scale of about $100 \mathrm{~s}$ [14]. The access to the relevant thermo-kinetic parameter of a single TS curve may be achieved by assuming that the response arises from a Debye process. Obviously, this is a simplification as in a real situation there is always a distribution of retardation times associated to the relaxational process, that may depend on the TS experimental variables [48]. Assuming an anelastic response described by a Voigt-Kelvin model, the temperature dependence of the retardation time may be given by:

$\tau(T)=\frac{\varepsilon(T)}{\beta|d \varepsilon(T) / d T|}$

By numerical derivation of the $\varepsilon(T)$ data, Arrhenius plots may be depicted, using Eq. (9). Using this procedure on the TS data of the studied PMMA some Arrhenius plots are shown in Figure 7. Assuming an Arrhenius form for $\tau(T)$, due to the short range of $\tau$ that are acceded (see data in Figure 7), the activation energy and the pre- 
N.M. Alves et al. / Polymer 45 (2004) 1007-1017

exponential factor may be calculated for each TS curve. More details on the calculation of thermokinetic parameters from TSR data may be found elsewhere [11].

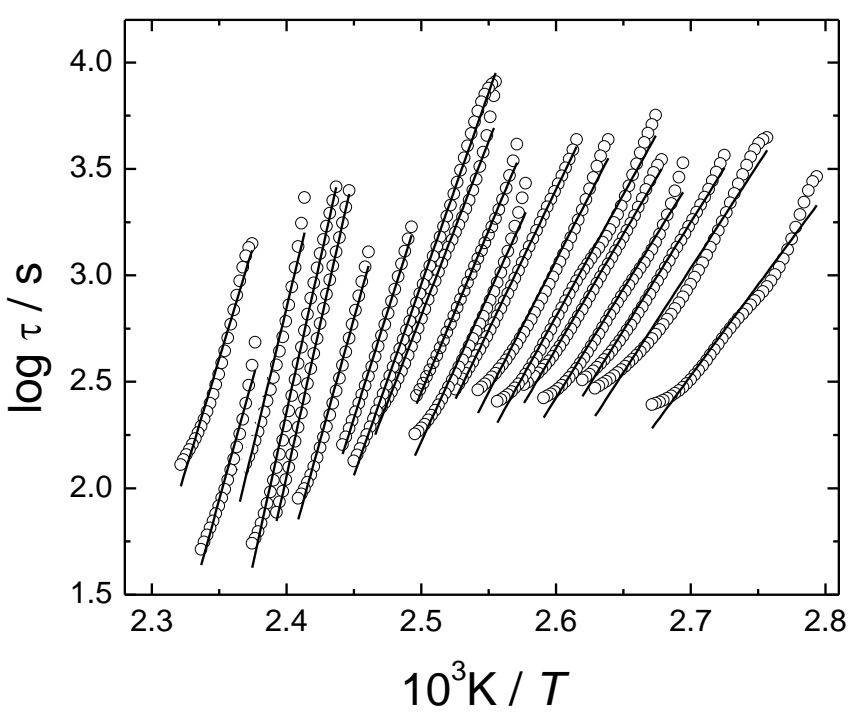

Figure 7 - Arrhenius plot of some TS experiments on the studied PMMA. The solid points were obtained using Eq. 9. The solid lines are the fitting with the Arrhenius equation.

It would be interesting to predict how TSR information could be compatible with usual DMA results, such as the one presented in section 3.1. Figure 8 shows a general Arrhenius diagram of an amorphous material near its glass transition. The typical curve of the main characteristic time $\langle\tau\rangle$ is shown, where the WLF behaviour at higher temperature gives rise to an Arrhenius tendency at lower temperatures. The thinner lines that envelop the $\langle\tau\rangle$ line pretend to represent a higher and a lower limit for the distribution of characteristic times. Both lines proceed along the reciprocal temperature axis approximately at the same vertical distance from the main $\langle\tau\rangle$ line, as a thermorheologically simple system is assumed. 


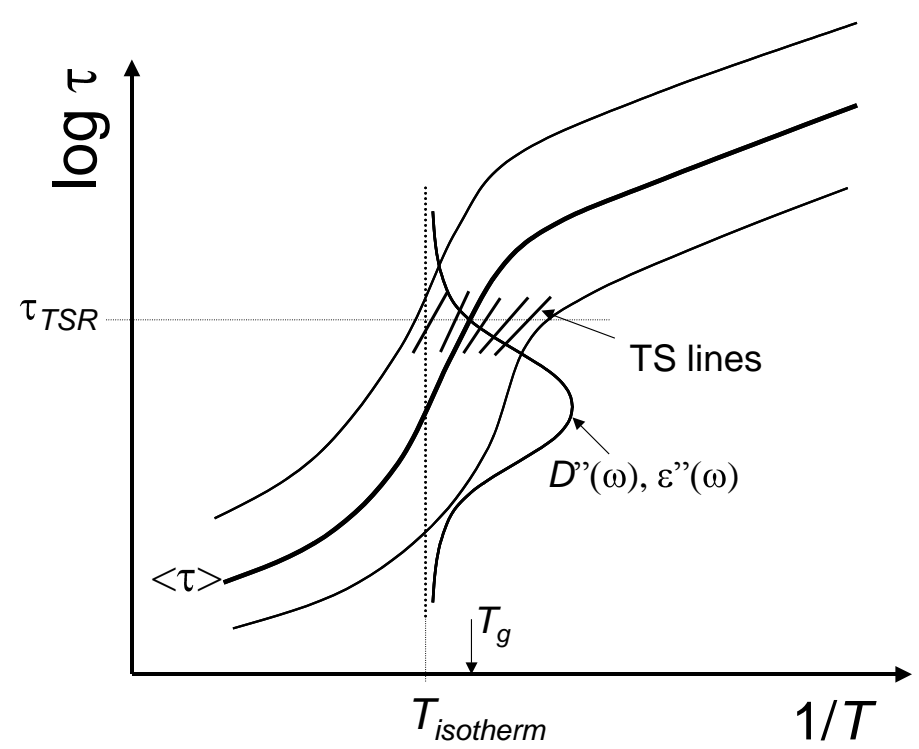

Figure 8 - The main curves are the typical profile of the retardation (characteristic) times in the glass transition region, showing the typical curvature at higher temperature (WLF-like) that tends to an Arrhenius behaviour below $T_{g}$. The thicker line is for the mean retardation (characteristic) time $(\langle\tau\rangle)$ and the two thinner lines (above and below the main line) represents an envelope of the distribution of characteristic times. The series of segments represents TS lines obtained at different $T_{\sigma}$, that covers a small time-range, around $\tau_{T S R}$. The peak along the $\log \tau$ axis represents schematically an isothermal DMA or dielectric experiment, where $D$ " or $\varepsilon$ " are plotted against $-\log \omega$, at a temperature above $T_{g}$.

A isothermal dynamic experiment (either a DMA or a dielectric relaxation spectroscopy, DRS, essay) is represented in Figure 8. The experiment was carried out at $T_{\text {isotherm }}$ and $D$ " or $\varepsilon$ " is plotted against $\log \tau$. A peak is observed with a maximum at about $\tau=\left\langle\tau\left(T_{\text {isotherm }}\right)\right\rangle$. Note that this peak is the result of the relaxation of all the retardation (characteristic) times active at $T_{\text {isotherm. }}$. TS experiments are also shown in this scheme, as a series of segments that correspond to Arrhenius lines obtained at different $T_{\sigma}$, going from the glassy to the liquid regions. Note that such experiments probe slower molecular motions (higher $\tau$ ) relatively to typical dynamic experiments, at the same temperature. Moreover, a thinner distribution of characteristic times is monitored, also with respect to isothermal DMA or dielectric experiments. In fact a TS experiment isolates the retardation times that at $T_{\sigma}$ are around $t_{\sigma}$, as those that are longer are not activated at the creep stage and the shorter ones are relaxed at the isothermal recovery stage, at $T_{\sigma^{-}} \Delta T_{w}$. Therefore, as $T_{\sigma}$ increases, from the glassy to the liquid states, TS curves are probing narrow distributed molecular motions at a time scale similar to that of a DSC scan (typically $\sim 100 \mathrm{~s}$ for an heating rate of $10{ }^{\circ} \mathrm{C} \cdot \mathrm{min}^{-1}[49]$ ), scanning the broad distribution of retardation times in an horizontal way (assuming the scheme of Figure 8), rather than the vertical scanning of an isothermal dynamic tests. The advantage of this horizontal inspection is that it is done in discrete steps: one may find, for example, 
N.M. Alves et al. / Polymer 45 (2004) 1007-1017

the apparent activation energy $E_{a}$ as a function of $T_{\sigma}$ for a nearly constant frequency [14]. The scheme of Figure 8 suggest that in the liquid state the slope of the TS lines (proportional to $E_{a}$ ) should increase as $T_{\sigma}$ decrease, i.e. for TS curves going to lower $1 / T$ values. However, near $T_{g}$, further decrease of $T_{\sigma}$ should lead to a decrease of $E_{a}$. This was in fact what it was observed by TSR in semi-crystalline poly(ethylene terephthalate) [12], and also by TSDC, in two liquid crystalline polymers [50]. Also in the present work the same trend was observed for the studied PMMA, as it is shown in Figure 9 (circles).

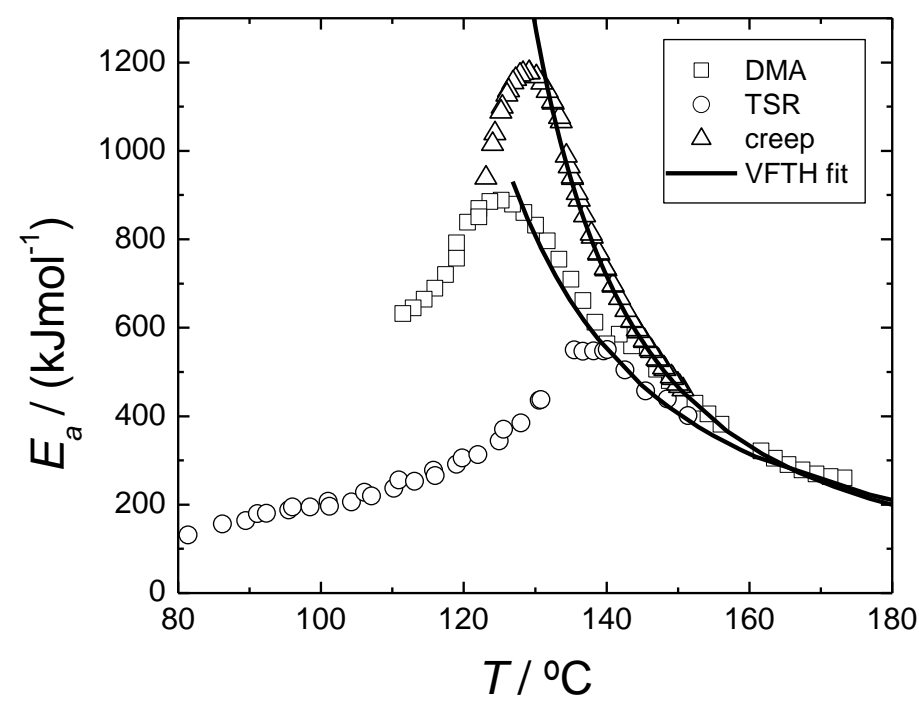

Figure 9 - Temperature dependence of the apparent activation energy across the glass transition of the studied PMMA. The squares are for the DMA results, calculated from the temperature shift factors (numerical differentiation). The fitting of these shift factors in the liquid state with the WLF equation also allowed to build a $E_{a}(T)$ curve with the corresponding $C_{1}$ and $C_{2}$ parameters (solid line). The circles are for the TSR results, where $E_{a}$ was obtained from the TS curves using Eq. 9 and assuming an Arrhenius behaviour (Figure 7).

In the case of DMA and creep experiments the apparent activation energy was obtained from the derivative of the experimental shift factor shown in Figure 5, which should also be similar to the value obtained from the central characteristic time $\langle\tau\rangle$,

$E_{a}(T)=R \ln (10) \frac{d \log a_{T}}{d(1 / T)}=R \frac{d \ln <\tau>}{d(1 / T)}$ 
N.M. Alves et al. / Polymer 45 (2004) 1007-1017

$E_{a}(T)$ goes through a maximum in the point at which the behaviour start deviating from the equilibrium line. Figure 9 shows the lines corresponding to DMA and creep results, this kind of plot can be compared with the same representation used for dielectric properties of different polymers [51].

The apparent activation energy can be obtained by TSR at quite low temperatures, far from $T_{g}$. In this temperature range $E_{a}$ depends only slightly on temperature, adopting an Ahrrenius behaviour characteristic of the glassy state. $E_{a}$ increases with increasing temperatures, going through a maximum when approaching to the VFTH regime, in the equilibrium liquid region at temperatures above $T_{g}$. But this approach occurs at temperatures clearly higher than in the case of the characteristic times of the modulus or the compliance. As a consequence the maximum value of $E_{a}$ is smaller when determined by TSR because in the VFTH regime $E_{a}$ is a decreasing function of temperature. The reason for this difference comes from the fact that in TSR the measurement is conducted on heating while creep and DMA experiments are isothermal. The behaviour in TSR is analogous to that found in DSC scans conducted on heating after subjecting the sample to a previous thermal treatment that ends at a low temperature, in the glassy state. The heat capacity measured in the heating scan starts with values characteristic of the glassy state $c_{p g}(T)$. The low molecular conformational mobility is responsible for these values of the heat capacity. During heating the glassy response of the material continues until temperatures higher than would be expected from a cooling scan. In other words the glass transition temperature interval shifts towards higher temperatures in the heating scan with respect to the cooling scan. This shift is highly dependent on the previous thermal history of the sample [52,53]. In the TSR experiments the transition from the Ahrrenius behaviour characteristic of the glass to the VFTH line characteristic of the equilibrium liquid is shifted towards higher temperatures with respect to the data obtained in isothermal experiments. Obviously, such conclusions could be extended to other thermally stimulated techniques such as TSDC.

Above $T_{g}$ the $E_{a}$ values obtained from DMA, creep and TS experiments agree very well. This is due to the fact that if the system is thermorheologically simple the slope of $\log \langle\tau\rangle v s 1 / T$ (from the DMA data) is similar to the slope of the TS curves in an Arrhenius diagram obtained at the same temperature, although at higher times (see Figure 8).

\subsection{Fragility}

As discussed before, fragility is related to the magnitude of the decrease of $\log \tau$ (or $\log a_{T}$, if one deals with rheological data) with decreasing $T_{g} / T$ and thus may be parameterised by the steepness "index" $m$ :

$$
m=\left.\frac{d \log \tau}{d\left(T_{g} / T\right)}\right|_{T=T_{g}}=\left.\frac{d \log a_{T}}{d\left(T_{g} / T\right)}\right|_{T=T_{g}}
$$


N.M. Alves et al. / Polymer 45 (2004) 1007-1017

The value $m=16$ corresponds to Arrhenius behaviour ("strong" limit) and for $m>200$ the systems reach the "fragile" limit [54]. The steepness index of many different materials are reported in some works [6,54,55]. Usually polymers appear at the fragile extreme of the Angell plot [54], although one can find also strong polymers [56,57]. It was found that $m$ (that gives the deviation from the Arrhenius behaviour) could be correlated with the non-Debye (or nonexponential) behaviour (often parameterised by the stretched exponential $\beta$ ), for a series of glass-forming liquids [54].

Using Eqs. 4 and 5, $m$ may be directly obtained from the VFTH or WLF parameters:

$$
m=\frac{B T}{\left(T_{g}-T_{0}\right)^{2}}=\frac{T_{g} C_{1}}{C_{2}}
$$

The usual value found for $C_{2}(\approx 50 \mathrm{~K})$ indicates that polymers with higher $T_{g}$ values tend to be the more fragile, which is the case of PMMA (of course there are counter examples).

Note that $m$ may be also obtained directly from the $E_{a}(T)$ plot, such as the one shown in Figure 7 (calculated with Eq. 10) from:

$m=E_{a}\left(T_{g}\right) /\left[\ln (10) R T_{g}\right]$

However fragility has both relaxational and thermodynamic manifestations and $m$, as defined before, only characterises the relaxational behaviour. In recent works $[6,7,58]$ it is frequent to distinguish between the socalled dynamic or kinetic fragility, measured by $m$, and the thermodynamic fragility quantified in several ways: usually by the step change on the heat capacity $\Delta C_{p}\left(T_{g}\right)$ [58] or by the ratio between the absolute heat capacities in the liquid and glassy states $\left(C_{p}^{l} / C_{p}^{g}\right)$ [6], or also by changes on the coefficient of thermal expansion [59] or on the excess entropy [60]. In all cases these variations are calculated at $T_{g}$.

As suggested by the Adam-Gibbs theory kinetically fragile liquids are expected to have large configurational heat capacities, resulting from their configurational entropy changing rapidly with temperature and kinetically strong liquids are expected to have small configurational heat capacities. For small molecule glasses this statement seems to be valid [61-63] but for polymers this is not so straightforward $[57,64,65]$. There are several works where it is shown that thermodynamic and kinetic fragilities are not strongly correlated $[65,66]$ especially for polymeric systems. This means that a kinetically fragile system is not necessarily a thermodynamically fragile system.

DSC can be used to study kinetic fragility. In this case the apparent activation energy around $T_{g}$, usually called $\Delta h^{*}[49,67]$, may be obtained from the variation of the fictive temperature with the cooling rate, $q_{c}$, in DSC scans according to $[29,49]$ 
N.M. Alves et al. / Polymer 45 (2004) 1007-1017

$\frac{d \ln q_{c}}{d\left(1 / T_{f}\right)}=-\frac{\Delta h^{*}}{R}$

$T_{f}$ is the fictive temperature defined as the temperature at which the structure of the glass would be in equilibrium if instantaneous brought to it, i.e., the temperature at which the value of an intensive property in the supercooled liquid is the one corresponding to the glass. Similarly to Eq. (13) the fragility index may be calculated with $m=\Delta h^{*} /\left[\ln (10) R T_{g}\right]$.

Different DSC traces obtained at $10^{\circ} \mathrm{C} \cdot \mathrm{min}^{-1}$, after different cooling rates are shown in Figure 10. $T_{f}$ was calculated as the temperature corresponding to the point of intersection of enthalpy curves of the glassy and the liquid states [68]. The $\ln q_{c}$ versus $1 / T_{f}$ plot of the studied PMMA is shown in Figure 11. From the slope of the corresponding linear fitting we found $\Delta h^{*}=771.6 \mathrm{~kJ}_{\text {.mol}}{ }^{-1}$ that leads to $m=102$, a value which is similar to the one reported in literature $(m=103)$ for PMMA [69] also determined by DSC. It should be noticed that the use of DSC in the determination of $m$ may lead to great errors, especially in fragile materials, where the variation of $T_{f}$ with $q_{c}$ is less pronounced, introducing significant inaccuracy in $\Delta h^{*}$ determination.

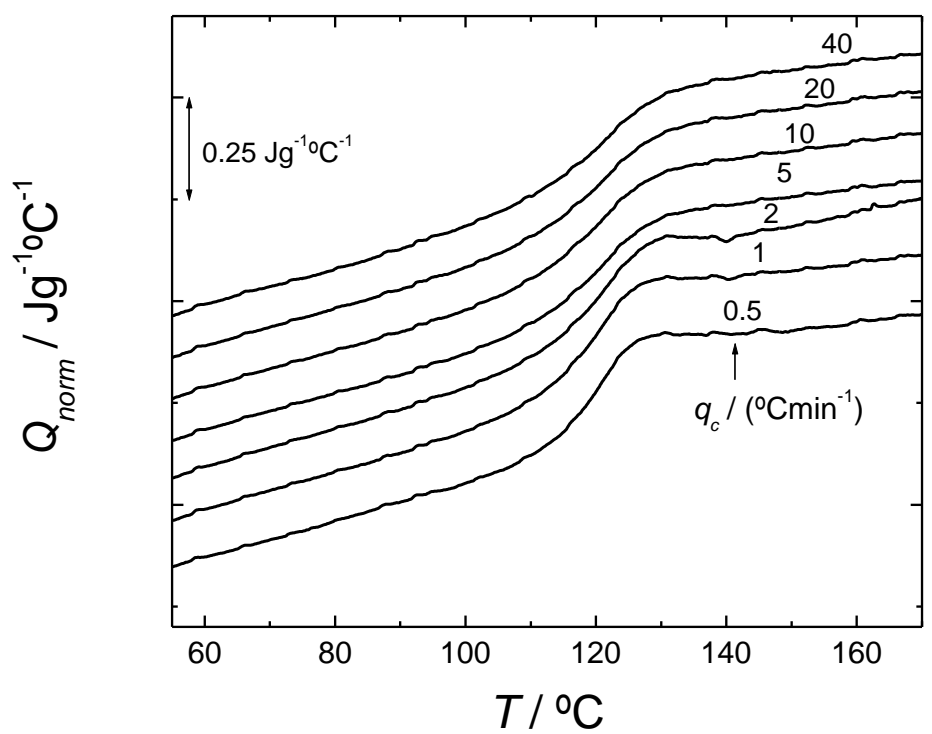

Figure 10 - DSC traces obtained for the studied PMMA at $10^{\circ} \mathrm{C} / \mathrm{min}$ after cooling from the liquid state at different rates $q_{c}=\mathrm{d} T / \mathrm{d} t$ (in the graphics). 


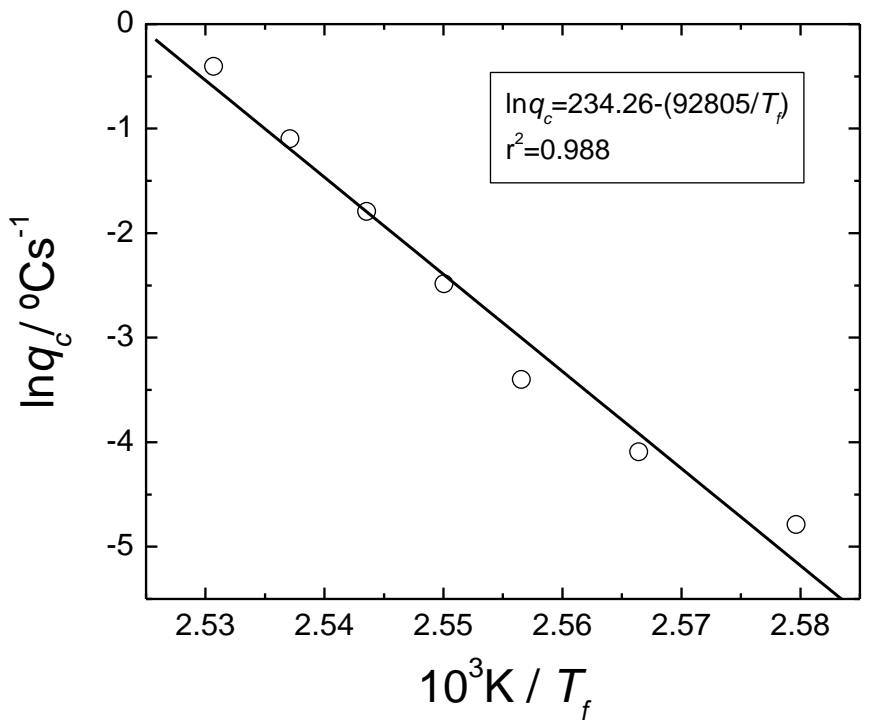

Figure 11 - logarithm of cooling rate $\left(\ln q_{c}\right)$ versus the reciprocal of fictive temperature $\left(1 / T_{f}\right)$ with the corresponding linear fitting, that allowed to calculate $\Delta h^{*}=771.6 \mathrm{~kJ} . \mathrm{mol}^{-1}$.

DSC was also used to evaluate the thermodynamic fragility and in this case we use the step change on the heat capacity $\Delta C_{p}$ at $T_{g}$. A value of $\Delta C_{p}\left(T_{g}\right)=0.21 \mathrm{~J} / \mathrm{gK}$ was obtained which is similar with the value found in literature for a PMMA: $\Delta C_{p}\left(T_{g}\right)=0.25 \mathrm{~J} / \mathrm{gK}$ [38], although our value is somewhat smaller as expected for a slightly crosslinked material. Comparing this value of $\Delta C_{p}$ with values found for other systems [38,58], this PMMA can be classified as a thermodynamically strong system a classification in agreement with the one found in reference [6] for PMMA, although they used the $\left(C_{p}^{l} / C_{p}^{g}\right)$ criterion. Angell suggested that this strong behaviour in polymers arises from the influence of chain length or entanglements [70], and in our sample the effect of entanglements is particularly pronounced. These two effects move $T_{g}$ to higher temperatures resulting in a smaller $C_{p}{ }^{l} / C_{p}{ }^{g}$ than the one predicted. From this point of view polymers should have a greater dynamic fragility than other materials and a smaller thermodynamic fragility. It is interesting to see how in reference [38] the authors observe for several systems the large values of $\Delta C_{p}\left(T_{g}\right)$ for the smaller $m$. However, as noted in reference [6] this explanation does not provide a complete picture of this behaviour since there are examples of polymers that fall in all the possible combinations of kinetic-thermodynamic fragility [6]. As noted by the authors this matter needs further investigation.

The concept of fragility has also been connected with the topology of the potential energy surface $[5,58]$ and in this context the energy minima available for the relaxation is not the same from one material to another. Thermodynamically $\Delta C_{p}\left(T_{g}\right)$ is proportional to this minima number [71].For the case of PMMA low variations 
N.M. Alves et al. / Polymer 45 (2004) 1007-1017

of $\Delta C_{p}\left(T_{g}\right)$ means that the accessible minima density is small and the material is thermodynamically strong. A high value of $m$ means that the average potential energy barrier is high and the material is kinetically fragile.

The high temperature dependence of the apparent activation energy in the VFTH equation makes the fragility parameter $m$ highly dependent of the criterion used to determine $T_{g}$. It is usual to define $T_{g}$ according to some ad hoc criterion, most of the times the temperature for which the relaxation time is 100 seconds. But the differences between the relaxation times measured by different techniques makes non sense a comparison of the absolute values of the fragility parameters determined with different techniques. The clear example is the difference between the viscoelastic relaxation and retardation times. The great difference between $D_{R}$ and $D_{U}$ (equation (3)), makes $\tau_{D}$ and $\tau_{E}$ to be several decades in time apart from each other. From our results it can be estimated that the temperature at which $\tau_{D}$ is $100 \mathrm{~s}$ is 15 degrees higher than that at which $\tau_{E}$ is $100 \mathrm{~s}$. A possible another criterion for $T_{g}$, which was chosen in this work, is the maximum of the $E_{a}(T)$ curve. With this assumption the DMA results would yield a value of $T_{g}(\mathrm{DMA})=125^{\circ} \mathrm{C}$ and using Equation (13) $\mathrm{m} \approx 117$ while creep experiments yield $T_{g}($ creep $)=128{ }^{\circ} \mathrm{C}$ and $\mathrm{m} \approx 153$. A value of $\mathrm{m} \approx 145$ was found by mechanical spectroscopy for PMMA in reference [6]. So, according to the fragility scale the PMMA network can be classified as a kinetically fragile system with a non-Arrhenius transport behaviour. As expected for fragile systems the apparent activation energy is high, usually fragile systems have $E_{a}$ of $500 \mathrm{~kJ} / \mathrm{mol}$ or more near $T_{g}$ (in our case $E_{a}$ is even higher), corresponding to a change in dynamics of one decade for a temperature change of $3-5 \mathrm{~K} \mathrm{[3]}$.

As explained above the maximum of the $E_{a}(T)$ curve corresponding to the TSR technique is shifted towards higher temperatures and lower $E_{a}$ values with respect to DMA and creep techniques are obtained, this obviously leads to a lower value of the fragility parameter $(\mathrm{m} \approx 70)$. It is interesting to note that the maximum value of $E_{a}$ obtained by TSR in this work is similar to the ones found in literature for PMMA by thermally stimulated creep $(\approx 590 \mathrm{~kJ} / \mathrm{mol})[72]$ and $\operatorname{TSDC}(\approx 350 \mathrm{~kJ} / \mathrm{mol})[73]$.

Also in other polymers lower values of $E_{a}\left(T_{g}\right)$ were obtained using thermally stimulated methods comparatively to the ones corresponding to other techniques (DSC, DMA, creep).For example, for polycarbonate and from TSDC results $E_{a}\left(T_{g}\right) \approx 317 \mathrm{~kJ} / \mathrm{mol}[74]$ and as $T_{g} \approx 158{ }^{\circ} \mathrm{C}$ (defined by the same criterion used in this work) $m$ will be $\approx 40$, but by mechanical spectroscopy $m \approx 132$ [54] which implies a much higher $E_{a}\left(T_{g}\right)$ value, and for amorphous PET and from TSDC results $\mathrm{Ea}\left(T_{g}\right) \approx 457 \mathrm{~kJ} / \mathrm{mol}[75]$ and $T_{g} \approx 69^{\circ} \mathrm{C}$ that will lead to a $m \approx 70$, but by DSC a value of $m \approx 156$ was obtained [6]. So the lower values of $m$ obtained by TSR (and TSDC) comparatively to the ones obtained by other techniques for the same material seem to be a consequence of the particular thermal profile of this technique.

\section{Conclusions}

In the case of the PMMA network studied in this work, DMA ( $E^{\prime}$ and $\tan \delta$ ) and creep compliance master curves were successfully obtained by simple shifting. For these techniques the shift factors above $T_{g}$ where well described by the WLF equation: Below $T_{g}$ a deviation from the Vogel behaviour was observed and the Arrhenius 
N.M. Alves et al. / Polymer 45 (2004) 1007-1017

diagram adopted a linear behaviour. The change from the Vogel to an Arrhenius behaviour was explained by the temperature dependence of the configurational entropy.

From the $\log a_{T}$ vs $1 / T$ representation it was clearly seen that at temperatures above $T_{g}$ the temperature dependence of the retardation times calculated from creep results and the temperature dependence of the relaxation times calculated from DMA results was not the same. This behaviour is the opposite of what is found in other materials and was attributed to the superposition of the $\alpha$ and $\beta$ relaxations of PMMA. In PMMA the $\beta$ peak plays an important role being a very large relaxation and in addition at high temperatures (above $T_{g}$ ) it is impossible to separate these two relaxations because they originate a new process - the $\alpha \beta$ process. We supposed that the influence of the $\beta$ relaxation would be distinct for the creep compliance and elastic modulus curves, being smaller in the case of the creep compliance curve which would lead to a distinct temperature dependence of the retardation and the relaxation times.

From TSR it was possible to obtain the apparent activation energy at low temperatures far from $T_{g}$, an information not easily available from DMA or creep techniques. In this temperature range $E_{a}$ depends only slightly on temperature adopting an Arrhenius behaviour. As the TSR experiments were conducted on heating while DMA and creep were isothermal, the change from an Arrhenius to a Vogel behaviour was detected at higher temperatures for TSR and the associated $E_{a}$ value was lower. Above $T_{g}$ the $E_{a}$ values obtained from DMA, creep and TSR experiments agreed very well.

Finally, the fragility of this PMMA network was also investigated. It should be pointed that the fragility index, $m$, is highly dependent of the criterion used to determine $T_{g}$. In our case $T_{g}$ was defined as the temperature of the maximum of the $E_{a}(T)$ curve. From DMA $m=117$ and from creep $m=153$. $m$ was also calculated from DSC results and a value of $m=103$ was obtained. So according to these results the PMMA network could be classified as kinetically fragile system. These values are in agreement with the ones found in literature for PMMA. By TSR lower values of $m$ would be obtained due to the particular thermal profile of this technique as explained before. DSC was also used to evaluate the thermodynamic fragility and in this case we adopted the step change on the heat capacity $\Delta C_{p}$ at $T_{g}$ criterion. A value of $\Delta C_{p}\left(T_{g}\right)=0.21 \mathrm{~J} / \mathrm{gK}$ was obtained. Comparing this value with the values found for other systems in literature we classified this PMMA network as a thermodynamically strong system.

\section{Acknowledgements}

NMA wishes to acknowledge Fundação para a Ciência e Tecnologia for the financial support through the grant PRAXIS XXI/BD/20327/99 
N.M. Alves et al. / Polymer 45 (2004) 1007-1017

References

[1] F.H. Stillinger, Science 267, 1935 (1995)

[2] C.A. Angel, Science 267, 1924 (1995)

[3] M.D. Ediger, C.A. Angel, S.R. Nagel, J. Phys. Chem. 100, 13200 (1996)

[4] K.L. Ngai, J. Non-Cryst. Solids 275, 7 (2000)

[5] C.A. Angell, J. Non-Cryst. Solids 131-133, 13 (1991)

[6] D. Huang, G.B. McKenna, J. Chem. Phys. 114, 5621 (2001)

[7] J.L. Green, K. Ito, K. Xu, C.A. Angell, J. Phys. Chem. B 103, 3991 (1999).

[8] N.M. Alves, J.F. Mano, J.L. Gómez Ribelles, Macromolecular Symposia, 148, 437 (1999).

[9]N.M. Alves, J.F. Mano, J.L. Gómez Ribelles, Polymer, 42, 4173 (2001).

[10] N.M. Alves, J.F. Mano, J.L. Gómez Ribelles, Mat. Res. Innovat., 4, 170 (2001).

[11] N.M. Alves, J.F. Mano, J.L. Gómez Ribelles, J. Thermal Analysis and Calorimetry, accepted.

[12] N.M. Alves, J.F. Mano, J.L. Gómez Ribelles, Polymer, 43, 3627 (2002)

[13] J.M. Cuesta Arenas, J. F. Mano, J.L. Gómez Ribelles, Journal of Non-Crystalline Solids, accepted.

[14] J.F. Mano, Thermochim. Acta 332, 161 (1999).

[15] N.T. Correia, C. Alvarez, J.J Moura Ramos, Polymer 41, 8625 (2000)

[16] N.T. Correia, C. Alvarez, J.J. Moura Ramos, M. Descamps, J. Chem. Phys. 113, 3204 (2000)

[17] J.D. Ferry, Viscoelastic Properties of Polymers, 3rd edn., John Wiley \& Sons, Inc., New York (1980)

[18] D. J: Plazek, K. L. Ngai, Macromolecules, 24, 1222 (1991)

[19] D. J. Plazek, J. Polym. Sci., Polym. Phys. Ed., 6, 621 (1968)

[20] R. A. Pethrick, W. J. Davis, Polymer, 39, 255 (1998)

[21] M.L. Williams, R.F. Landel, J.D. Ferry, J. Am. Ceram. Soc. 1953; 77: 3701.

[22] H. Vogel, Phys. Z. 1921; 22: 645.

[23] G.A. Fulcher, J. Am. Chem. Soc. 1925; 8: 339.

[24] G. Tamman, W.Z. Hesse, Anorg. Allg. Chem. 1926; 156: 245.

[25] J. Batchinski, Z. Phys. Chem., Stoechiom. Verwandtschaftsl. 84, 643 (1993)

[26] R. J. Speedy, C. A. Angell, J. Chem. Phys. 65, 851 (1996)

[27] U. Bengtzelius, W. Götze, A. Sjölander, J. Chem. Phys. 17, 5915 (1989)

[28] R. Richert. H. Bassler, J. Phys. B Condensed Matter 2, 2273 (1990)

[29] T. A. Litovitz, J. Chem. Phys. 20, 1088 (1952)

[30] T. Hideshima, J. Japan, Soc. Testing Materials 10, 246 (1961)

[31] T. Hideshima, in "Solid Physics Vol. 14" Seitz and Turnbull Eds. Academic Press, New York (1963)

[32] P.R. Higgenbotham-Bertolucci, H. Gao, J.P. Harmon, Pol. Eng. Sci. 41, 873 (2001)

[33] A. Alegria, E. Guerrica-Echevarría, L. Goitiandía, I. Tellería, J. Colmenero, Macromolecules 28, 1516 (1995)

[34] S. Saito, J. Appl. Polym. Sci. 2, 93 (1959) 
N.M. Alves et al. / Polymer 45 (2004) 1007-1017

[35] E. Schlosser, Polymer Bulletin 8, 461 (1982)

[36] G. Adams, J.H. Gibbs, J. Chem. Phys. 43, 139 (1958)

[37] J. L. Gomez Ribelles,A Vidaurre Garayo, J. M. G. Cowie, R. Ferguson, S. Harris, I. J. McEwen, Polymer 40, 183 (1998)

[38] E.Hempel, G. Hempel, A Hensel, C. Schick, E. Donth, J. Phys. Chem. B 104, 2460 (2000)

[39] V. B: F. Mathot, Polymer 25, 579 (1984)

[40] K. L. Ngai, S. Mashimo, G. Fytas, Macromolecules 21, 3030 (1988)

[41] J. Heijboer, Proc. Int. Congr. Phys. Non-Cryst. Sol., Delft (1964)

[42] E. Muzeau, J. Perez, G. P. Johari, Macromolecules 24, 4713 (1991)

[43] N. G. McCrum, B. E. Read, G. Williams, Anelastic and Dielectric Effects in Polymeric Solids, Dover Publications Inc., New York (1967)

[44] G. Williams, Adv. Polym. Sci. 33, 59 (1979)

[45] G. Williams, Trans. Faraday Soc. 62, 2091 (1966)

[46] G. Williams, D. C. Watts, in Dielectric Properties of Polymers, F. E., Karasz (Ed.), Plenum Press, New York, 1972

[47] F. Garwe, A, Schönhals, H. Lockwenz, M. Beiner, K. Schröter, E. Donth, Macromolecules 29, 247 (1996)

[48] N.M. Alves, J.F. Mano, J.L. Gómez Ribelles, Polym. Int., 51, 434 (2002)

[49] C.T. Moynihan, A.J. Easteal, M.A. deBolt, J. Tucker, J. Am. Ceram. Soc. 59, 12 (1976)

[50] J.J. Moura Ramos, J.F. Mano, D. Coates, Mol. Cryst. Liq. Cryst. 281, 267 (1996)

[51] J. L. Gómez Ribelles, R. Diaz Calleja, Anales de Fisica, Serie B 81, 104 (1985)

[52] A R. Berens, I. M. Hodge, Macromolecules 15, 756 (1982)

[53] A R. Berens, I. M. Hodge, Macromolecules 18, 1980 (1985)

[54] R. Böhmer, K.L. Ngai, C.A. Angell, D.J. Plazek, J. Chem. Phys. 99, 4201 (1993)

[55] E. Rössler, K.-U. Hess, V.N. Novikov, J. Non-Cryst. Solids 223, 207 (1998)

[56] C. M. Roland, Macromolecules 25, 7031 (1992)

[57] P. G. Santangelo, K.L. Ngai, C. M. Roland, Macromolecules 29, 3651 (1996)

[58] A. Saiter, C. Devallencourt, J. M. Saiter, J. Grenet, Euro. Polym. J. 37, 1083 (2001)

[59] D. M. Colluci, G. B. Mckenna, J. J. Filliben. A. Lee, D. B. Curliss, k. B. Bowman, J. D. Russel, J. Polym Sci. Polym. Phys. Ed. 35, 1561 (1997)

[60] K. Ito, C. T. Moynihan, C. A. Angell, Nature 398, 493 (1999)].

[61] C.A Angell, Science 67, 1924 (1995)

[62] K. L. Ngai, D. J. Plazek, Rubber chem. Technol. 68, 376 (1995)

[63] C. A Angell. B. E. Richards, V. Velikov, J. Phys.: Condens. Matter 11, A75 (1999)

[64] K. L. Ngai, C. M. Roland, Macromolecules 26, 6824 (1993)

[65] C. M. Roland, P. G. Santangelo, K. L. Ngai, J. Chem. Phys. 111, 5593 (1999)

[66] G. P. Johari, J. Chem. Phys. 112, 8958 (2000) 
N.M. Alves et al. / Polymer 45 (2004) 1007-1017

[67] I.M. Hodge, J. Non-Cryst. Sol. 169, 211 (1994)

[68] A.Q. Tool, J. Am. Ceram. Soc. 29, 240 (1946)

[69] E. Donth, J. Polym. Sci. Phys. Ed. 34, 2881 (1996)

[70] C. A Angell, in Structure and Properties of Glassy Polymers, M. R. Tant and A J. Hill (Eds.), American Chemical Society, Washington DC, 1998, chap. 3, pp. 37-52

[71] C. A Angell, J. Phys. Chem Solids 49, 963 (1988)

[72] S. Doulut, P. Demont, C. Lacabanne, Macromolecules 33, 3425 (2000)

[73] B. B. Sauer, P. Avakian, Polymer 33, 5128 (1992)

[74] A Bernes, D. Chatain, C. Lacabanne, Polymer 33, 4682 (1992)

[75] E. Dargent, M. Kattan, C. Cabot, P. Lebaudy, J. Ledru, J. Grenet, J. Appl. Polym. Sci. 74, 2716 (1999) 